\title{
La peau : un autre modèle pour étudier la formation des profils morphogénétiques chez les vertébrés
}

La peau des vertébrés est un organe segmenté. Si cette affirmation peut venir à l'esprit lors de l'observation des rangées d'écailles d'un reptile ou des chevrons de plumes d'un oiseau, elle semble à première vue difficile à appliquer à l'espèce humaine. Pourtant, les dermatologistes connaissent de nombreux exemples de dermatoses qui se développent de façon symétrique sur les régions droite et gauche du corps, selon des chemins qui semblent préétablis depuis l'embryogenèse.

La peau est formée de deux tissus, un épithélium, l'épiderme, et un tissu conjonctif, le derme, qui ont une origine embryologique différente. Des expériences de transplantations hétérospécifiques caille-poulet, réalisées à 2 jours d'incubation, montrent de plus que la formation de la peau de la région céphalique diffère de celle du reste du corps. L'épiderme provient de l'ectoderme, sauf dans la région nasofrontale, où il est originaire de la partie la plus antérieure des bourrelets neuraux [1]. Le derme, quant à lui, a quatre origines différentes (figure 1a). Celui de la région dorsale de la tête se forme à partir du mésoderme paraxial dit insegmenté dès le niveau de la placode otique (ou somitomères) ; le derme de la face provenant des crêtes neurales [2, 3]. Le derme des membres et de la face ventrale du corps provient du mésoderme somatopleural latéral, alors que celui de la région dorsale est formé à la suite de la migration des cellules du dermatome des somites [4]. Nous voyons donc apparaître par deux fois la notion de segmentation au cours de la formation de la peau : celle des crêtes neurales, correspondant aux neuromères dont elles sont issues, et celle des somitomères, ou celle, plus évidente, des somites.
$\mathrm{Si}$ la peau des vertébrés était un organe plan, constitué simplement par la superposition de deux tissus (ce qui est réalisé dans le cas de certaines mutations), son étude serait de peu d'intérêt pour notre propos. En fait, elle va former au cours du développement embryonnaire et postnatal ce que l'on a coutume d'appeler des annexes tégumentaires. Celles-ci appartiennent à deux grandes catégories : les phanères (écailles des reptiles, plumes des oiseaux, poils des mammifères) et les glandes cutanées (particulièrement diversifiées chez les mammifères). Phanères et glandes cutanées ont, d'une part, une morphologie qui leur est propre, et sont, d'autre part, répartis de façon ordonnée ; l'architecture précise et le motif de distribution de chaque type d'annexe étant caractéristique pour une espèce donnée de chaque région du corps [5]. La morphogenèse du tégument implique donc la mise en jeu des deux types de profils morphogénétiques : agencement des cellules entre elles pour construire une structure simple, telle qu'une écaille, ou plus complexe, telle qu'une plume, et répartition de ces différentes structures dans la peau. Si le derme semble jouer un rôle de premier plan, notamment en ce qui concerne la distribution des annexes et l'achèvement de leur morphogenèse, celui de l'épiderme ne doit pas être sous-estimé : l'ectoderme dont il est issu pouvant présenter une régionalisation de sa compétence à former certains types d'annexe.

De nombreuses expériences ont montré que le derme détient et transmet à l'épiderme l'information relative à la distribution des placodes, premières ébauches tégumentaires, formées par un épaississement localisé de l'épiderme. Par exemple, l'association de derme de lèvre supérieure d'embryon de souris (figure $2 a, p$. 860) et d'épiderme dorsal de souris, ou bien encore d'embryon de poulet ou de lézard [5], aboutit à la formation d'ébauches de phanères disposées comme des vibrisses. Ce type d'information, qui a conservé un support similaire au cours de l'évolution des vertébrés, serait déjà contenu dans les cellules des crêtes neurales avant leur migration. En effet, chez l'embryon d'oiseau la greffe du territoire présomptif de la première crête neurale à la place de celui de la troisième provoque la formation d'un cercle de plumes surnuméraire, typique de l'orifice du conduit auditif dans cette classe [3]. Un type d'information d'ordre comparable, requis pour l'arrangement des plumes des différentes zones de la ptéryle spinale (dorsale) des oiseaux (figure 1a) est également le propre des cellules du dermatome avant leur migration [4]. Or, les travaux de l'équipe de D. Duboule ont montré que chaque point de l'axe céphalo-caudal des vertébrés, de la région occipitale à la région caudale, était défini par un pourcentage d'expression d'un certain nombre de gènes à homéoboîte, s'emboîtant comme des poupées russes [6]. Notons que l'un de ces Hox, le 4.4, a été mis en évidence dans les cellules dermiques au moment de la formation des placodes plumaires chez l'embryon de poulet [7]. La limite antérieure de répartition des gènes $H o x$ ne dépasse pas le deuxième arc branchial figure 1b). Récemment, de nouveaux gènes à homéoboîte, notamment Emx2 et Dlx-1, ont été isolés chez la souris, par analogie avec les gènes empty spiracle [8] et distal-less [9] de la drosophile. La répartition de leur expression est principalement céphalique (figure $1 b$ ). La région céphalique se distinguerait donc du reste du corps par l'emploi d'un système différent de gènes du développement pour engendrer sa diversité régionale. Nous n'avons encore aucune 


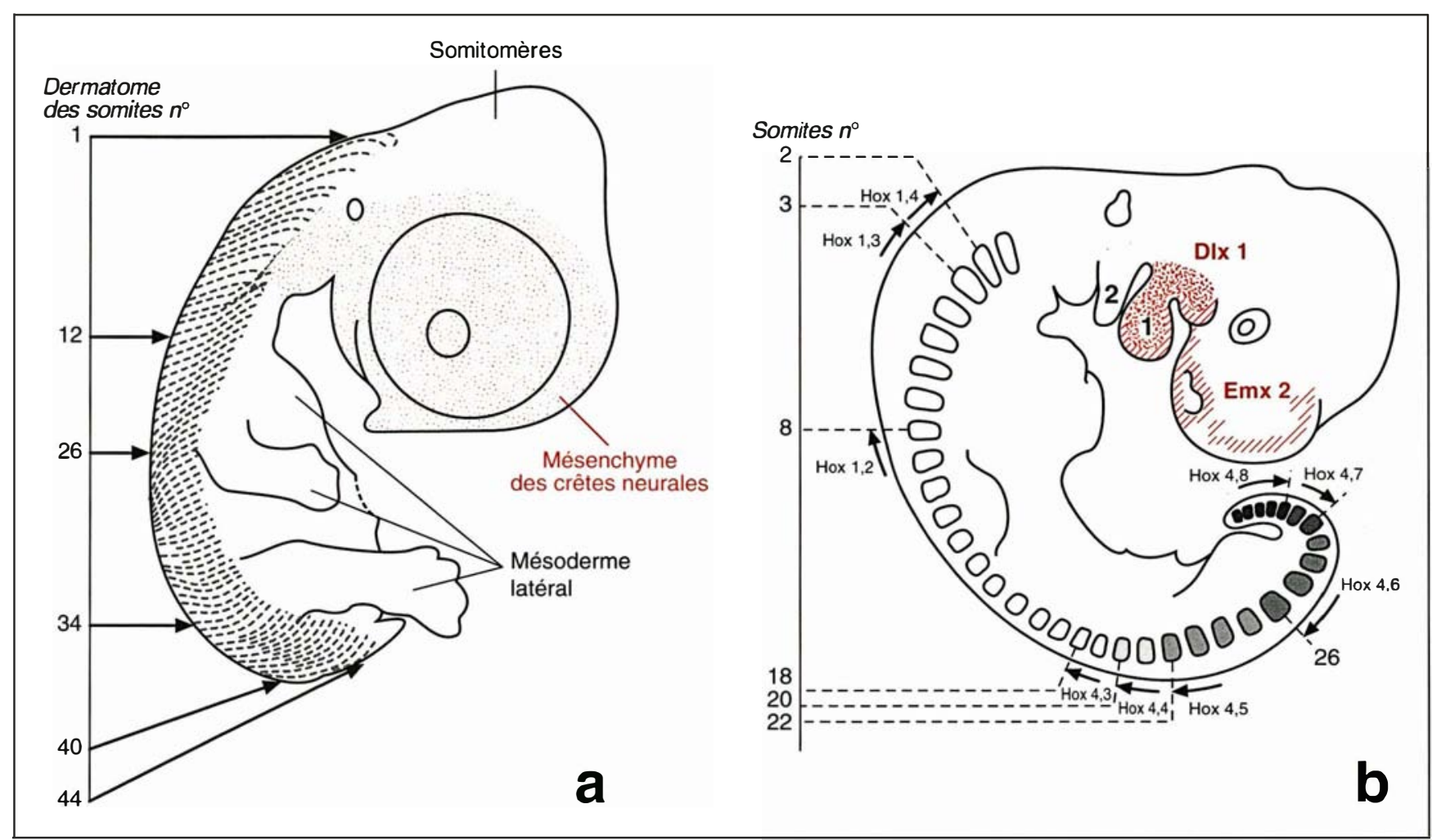

Figure 1. Comparaison de l'origine des différents territoires du derme de la peau d'un embryon de poulet de 6,5 jours d'incubation (a) et de la répartition de l'expression de quelques gènes à homéoboîte chez l'embryon de souris de 10,5 jours de gestation (b). Chez l'embryon de poulet, le derme de chaque territoire de la région dorsale est formé par la migration de cellules issues d'une catégorie particulière de somites: par exemple, le derme de la face dorsale du cou correspond aux somites 1 à 12 [4], alors que celui de la face provient des crêtes neurales [2]. Chez l'embryon de souris, les limites antérieures de l'expression somitique des gènes Hox 1 et Hox 4 sont indiquées par des flèches. Notez que le domaine d'expression des gènes Hox 4 ne dépasse pas les somites lombaires [28]. Le niveau d'expression céphalique des gènes Dlx-1 [9] et Emx2 [8] sont respectivement indiqués par des pointillés et des hachurés rouges. (N.B. : l'expression de ces différents gènes au niveau des bourgeons de membre n'a pas été indiquée sur le schéma.) 1 : arc mandibulaire; 2 : arc hyoïdien.

preuve expérimentale que les gènes à homéoboîte interviennent réellement dans la spécification de la répartition des annexes tégumentaires; alors que l'on sait que les gènes $H o x$ peuvent être réglés par des facteurs nucléaires induits par l'acide rétinoïque [10] et que l'on a montré que le traitement par l'acide rétinoïque, en modifiant leur distribution, respécifie l'identité des vertébrés [11].

Le derme dirige également la construction d'une annexe tégumentaire selon un plan régionalement défini. Par exemple (figure 2a), l'association de derme de lèvre supérieure d'embryon de souris et d'épiderme dorsal aboutit à la formation de follicules pileux de grand diamètre, présentant un étranglement " en sablier ", deux caractères $\mathrm{m} / \mathrm{s} n^{\circ} 8$, vol. 8, actobre 92 qui définissent un follicule de vibrisse. Normalement, la formation d'un tel follicule est précédée par l'expression des récepteurs nucléaires de l'acide rétinoïque (RAR) de types $\alpha$ et $\gamma$ [12]. La formation de glandes de type glomérulaire provoquée par le traitement par l'acide rétinoïque de lèvre supérieure d'embryon de souris [13] résulte de l'induction de l'expression du récepteur $\beta$ dans le derme [14]. Cependant, des résultats inédits de $\mathrm{J}$. Viallet dans mon groupe montrent que seul l'épiderme de lèvre supérieure est capable de répondre à la présence dermique de RAR $\beta$ en formant des glandes en lieu et place de vibrisses (figure $2 b$ ). Or cet épiderme céphalique est défini entre autres par l'expression du gène $E m \times 2$ [8] (figure 1b). Ainsi, le derme ne pour- rait diriger la construction d'une annexe tégumentaire donnée qu'à l'expresse condition que l'épiderme associé soit compétent, en d'autre termes se manifeste lui-même par l'expression d'un ensemble précis de gènes à homéboîte. Le rôle du composant ectodermique dans la morphogenèse du tégument n'est donc pas négligeable. Rappelons que le mésenchyme mandibulaire ne peut acquérir ses potentialités inductrices dentaires que s'il est associé à un stade précoce à un ectoderme de la face [15]. De même, l'origine régionale de l'ectoderme peut guider le choix entre la formation d'une plume ou d'une écaille chez l'embryon d'oiseau. Le corps d'un poulet est recouvert de plumes, sauf les pattes qui sont normale- 


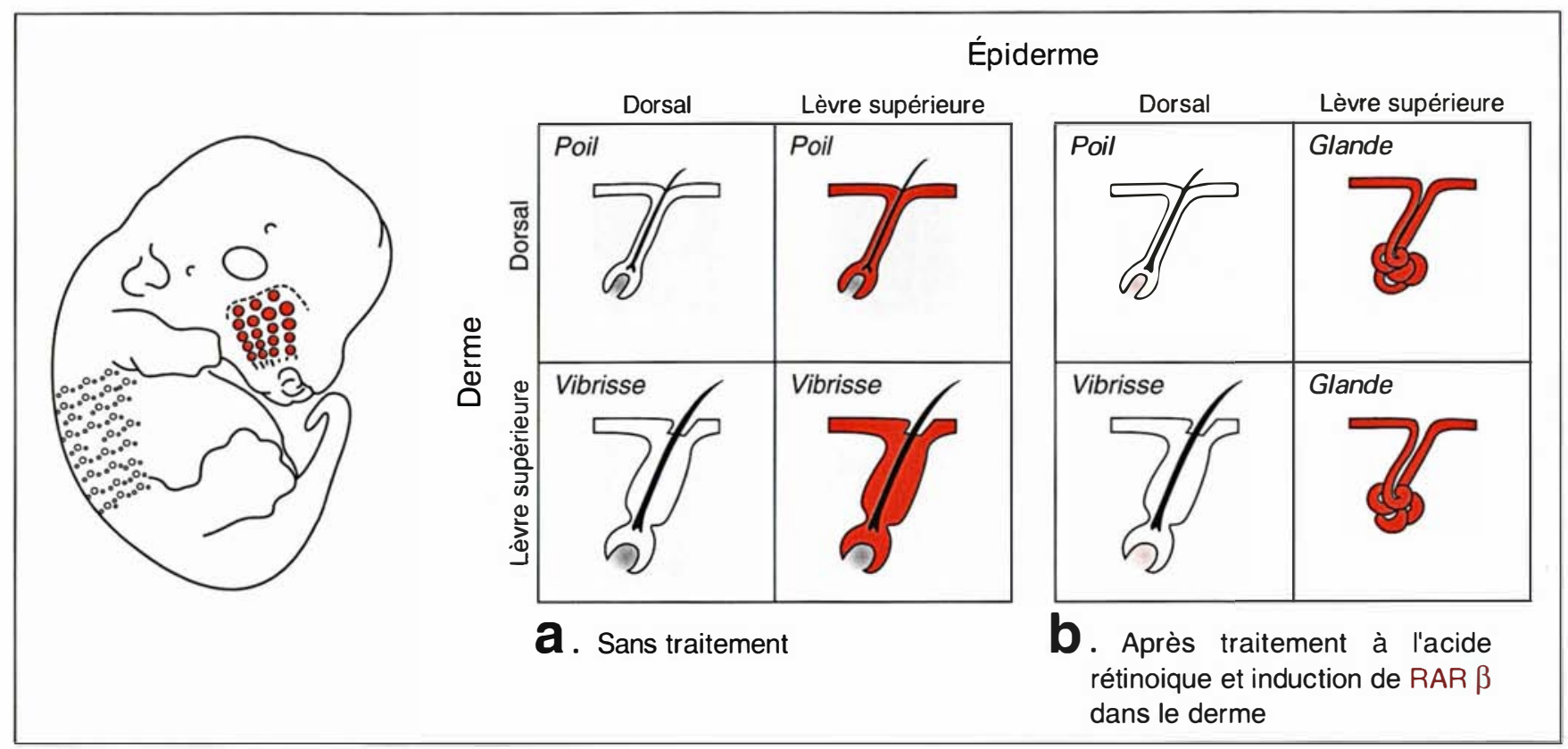

Figure 2. Rôles respectifs du derme et de l'épiderme lors de la formation des annexes tégumentaires chez l'embryon de souris. La peau de lèvre supérieure ou du dos est prélevée avant l'apparition des placodes. Elle est séparée en ses deux constituants, derme et épiderme, qui sont ensuite recombinés de façon homotopique ou hétérotopique, puis cultivés sur milieu normal (a) [5] ou additionné d'acide rétinoïque (b) [12-14]. Ce dernier induit dans le derme l'expression de son récepteur nucléaire $\beta(R A R \beta)$, récepteur qui n'est pas exprimé normalement de façon détectable au niveau du tégument. Seul l'épiderme de lèvre supérieure est capable de répondre à la présence de RAR $\beta$ dans le derme par la formation de glandes. Cependant, lors de la formation de follicules pileux, l'origine du derme détermine leur morphologie et leur distribution selon un motif de vibrisses ou de poils de pelage.

Crêtes neurales : ensembles de cellules neurectodermiques, situées au sommet des bourrelets neuraux, qui ne s'intègrent pas à la paroi du tube neural et qui, à la suite de migrations, vont donner différents types cellulaires, entre autres des cellules ganglionnaires, les cellules pigmentaires, des cellules endocrines, telles que celles de la médullosurrénale, ainsi que le squelette facial et le derme de la face et de la région anténieure $d u$ cou.

Mésoderme : feuillet médian embryonnaire se mettant en place au cours de la gastrulation entre le feuillet superficiel ou ectoderme (qui donnera en particulier l'épiderme) et le feuillet profond ou endoderme (qui donnera entre autres l'épithélium digestiff. Il se subdivise, chez l'embryon de vertébrés, en mésodernes axial, paraxial et latéral.

Mésoderme axial ou corde : comme son nom l'indique, cylindre plein situé dans l'axe céphalo-caudal de l'embryon, immédiatement sous le neurectoderme, qui s'organise en tube nerveux. Organe de soutien embryonnaire, il

\section{GLOSSAIRE}

sera peu à peu remplacé par l'édification des corps vertébraux.

Mésoderme paraxial : situé de part et d'autre de la corde. Il comprend les somites et les somitomères.

Somitomères : mésoderme paraxial présentant une amorce de segmentation.

Somites : mésoderme paraxial segmenté, le somite numéro 1 étant situé juste en arrière du niveau d'édification ultérieur de la placode otique (à l'origine de l'oreille). Les somites, petites masses épithéliales creuses, se subdivisent en quatre régions qui correspondent à quatre potentiels migratoires différents une région ventrale ou sclérotome, à l'origine des vertèbres ; deux régions latérales ou myotomes - qui vont former, d'une part, les muscles paravertébraux et intercostaux, et, d'autre part, les muscles du tronc et des mernbres - et enfin une région dorsale ou dermatome.

Dermatome : ensemble de cellules consti- twant la partie dorsale de chaque somite, qui, à la suite d'une migration, vont former le derme de la peau.

Mésoderme latéral : mésoderme situé de part et d'autre des somites. Il se délamine en doux feuillets, délimitant une cavité ou coelome. Le feuillet le plus interne ou mésoderme splanchnopleural donnera en particulier les muscles viscéraux; le feuillet le plus externe ou mésoderme somatopleural formera entre autres les os et les tendons des mernbres, ainsi que le derme de la face ventrale $d u$ corps, des flancs et des mernbres.

Neuromères : différents compartiments du tube nerveux anténieur.

Ptéryle spinale : ensemble de plumes situées dans la région dorsale du tégument des oiseaux, de la région occipitale à la base de la queue.

Ptéryle fémorale : ensemble de plumes situées sur le segment proximal de la patte des oiseaux. 
ment recouvertes d'écailles. Or il existe trois possibilités [16] (figure 3) d'obtenir des poulets à pieds emplumés. La première est une mutation présentée par plusieurs souches, telles que les Faverolles ou les Bantam de Pékin. La deuxième est de recombiner au stade du bourgeon de membre une calotte ectodermique de bourgeon d'aile et une pulpe mésodermique de bourgeon de patte [17]. La troisième est de traiter un embryon de poulet de 10 jours d'incubation (au moment où normalement doivent se former les premières ébauches d'écailles) par l'acide rétinoïque [18]. Des expériences de recombinaison de derme et d'épiderme de patte d'embryons traités ou non [19] montrent que, dans ce dernier cas, c'est la compétence de l'épiderme qui est changée. On peut interpréter de deux façons différentes la formation de pieds emplumés par le traitement à l'acide rétinoïque : soit l'on considère que le tégument de la patte est affecté dans sa régionalisation proximodistale, et l'on obtiendrait en quelque sorte des plumes fémorales sur le pied; soit l'on considère que le tégument de la patte est antériorisé en tégument d'aile. La présence de plumes de type vol (des rémiges) sur les pieds des poulets Bantam serait en faveur de la deuxième hypothèse. La formation de plumes sur les pieds du poulet, ou de glandes sur la lèvre supérieure de souris, sont des modifications de type homéotique provoquées par l'acide rétinoïque. Leur relation avec un changement d'expression de gènes à homéoboîte est en cours d'étude.

Des interactions réciproques entre le derme et l'épiderme sont donc responsables de l'établissement des profils morphogénétiques tégumentaires. Ces interactions résultent des propriétés de ces deux tissus, qui seraient fondées sur l'expression de gènes à homéoboîtes, pouvant être contrôlés par l'intermédiaire des ligands de l'acide rétinoïque, de type RAR [20-22], ainsi que par d'autres facteurs corégulateurs qui augmenteraient leur diversité. Mais quel est le support moléculaire des échanges d'informations entre les deux composants de la peau ? L'hypothèse du rôle joué par les différents composés de la membrane basale et de la matrice extracellulaire dermique [23] a été avancée à maintes reprises, de $\mathrm{m} / \mathrm{s} n^{\circ} 8$, vol. 8, actobre 92

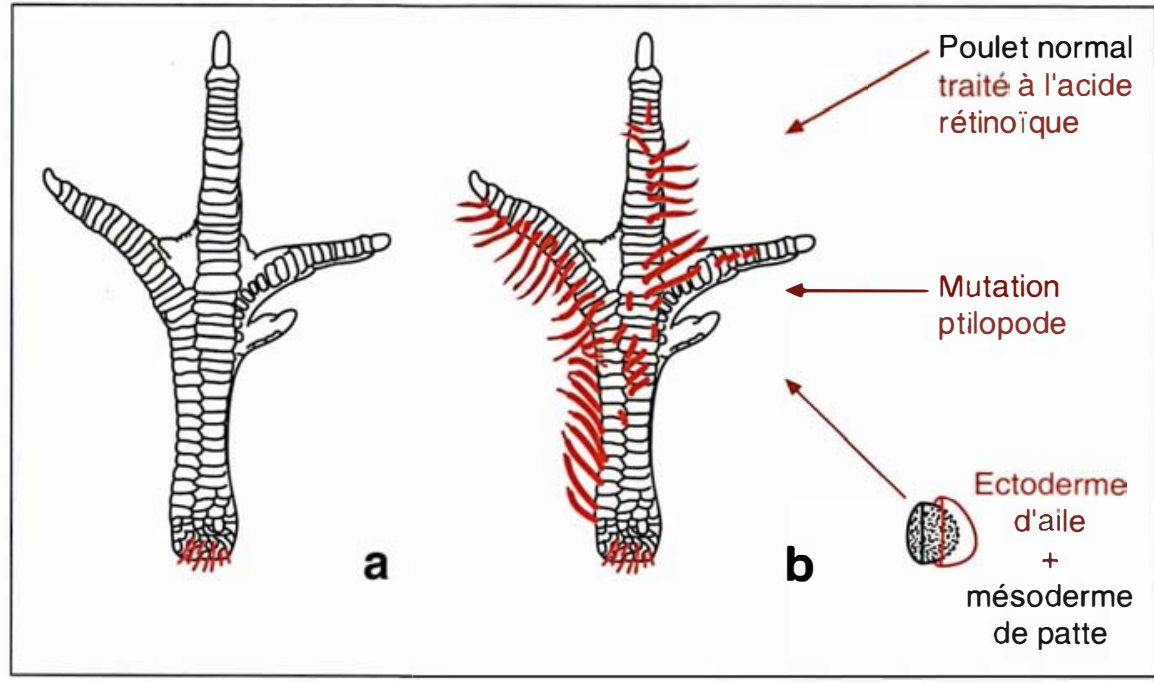

Figure 3. Trois possibilités d'obtention de pieds emplumés chez l'embryon de poulet. Le pied du poulet est normalement recouvert d'écailles (a). II existe une mutation, appelée ptilopode, qui provoque la formation de plumes sur les écailles (b). La formation de pieds emplumés peut également être obtenue par traitement à l'acide rétinoïque au stade de la formation des écailles [18] ou par recombinaison d'un ectoderme de bourgeon d'aile et d'une pulpe mésodermique de bourgeon de patte [17].

même que le rôle que pourraient jouer les molécules d'adhérence cellulaire [24, 25]. Cependant, si ces diverses molécules ont sans conteste un rôle dans la morphogenèse tégumentaire, et peuvent transmettre des informations par contact, d'autres protéines - qui, elles, peuvent être transférées d'un tissu à l'autre - devraient avoir un rôle primordial : celles appartenant à la superfamille des facteurs de croissance. Ce type de protéines est sous le contrôle de l'acide rétinoïque et des gènes à homéoboîte. Ainsi, l'ARNm et la protéine TGF $\beta 2$ sont détectés dans le derme de la peau dorsale de l'embryon de souris à 15 jours de gestation, au cours de la formation des bourgeons pileux, alors que la protéine seule est détectée peu après dans l'épiderme [27].

En conclusion, le rebond fantastique donné aux recherches actuelles en biologie du développement - grâce à la découverte des gènes à homéoboîte des vertébrés [6], de celle des récepteurs nucléaires de l'acide rétinoïque [20-22], ainsi que de celle de nombreux facteurs de croissance, tant épithéliaux que fibroblastiques - devrait permet- tre très rapidement, dans les années à venir, d'avancer de façon notable notre compréhension des mécanismes mis en jeu lors de l'établissement des profils morphogénétiques, et en particulier lors de la formation du tégument des vertébrés

Danielle Dhouailly

Jeune Equipe Biologie de la différenciation épithéliale, universilé Joseph-Fourier, CERMO, BP $53 X$, Grenoble Cedex, France.

\section{TIRÉS A PART}

D. Dhouailly.

\section{RÉFÉRENCES}

1. Couly G, Le Douarin N. The fate map of the cephalic neural primordium at the presomitic to the 3-somite stage in the avian embryo. Development 1988 ; 103s : 101-13.

2. Lc Lièvre C, Lc Douarin N. Origine ectodermique du derme de la face et du cou montrée par des recombinaisons interspécifiques chez l'embryon d'oiseau. CR Acad Sci $1974 ; 278$ : $517-20$.

$\rightarrow$ 
3. Noden DW. Interactions and fates of avian craniofacial mesenchyme. Development 1988 ; 103s : 121-40.

4. Mauger A. Rôle du mésoderme somitique dans le développement du plumage dorsal chez l'embryon de poulet. II Régionalisation du mésoderme plumigène. $J$ Embryol Exp Morph 1972 ; 28 : 343-66.

5. Dhouailly D. Dermo-epidermal interaction during morphogenesis of cutaneous appendages in amniotes. Front Matrix Biol $1977 ; 4$ : 86-121.

6. Duboule D, Dollé P. The structural and functional organization of the murine HOX gene family resembles that of Drosophila homeotic genes. EMBO J $1989 ; 8$ : 1497-505.

7. Chuong CM, Oliver G, Ting SA, Jegalian BG, Chen HM, De Robertis EM. Gradients of homeoproteins in developing feather buds. Development 1990 ; 110 : 1021-30.

8. Simeone A, Gulisano M, Acampora D Stornaiuolo A, Rambaldi M, Boncinelli E Two vertebrate homeobox genes related to the Drosophila empty spiracles gene are expressed in the embryonic cerebral cortex. EMBO J 1992 $7: 2541-50$.

9. Dollé P, Price M, Duboule D. Expression of the murine Dlx-1 homeobox gene during facial, ocular and limb development. Differentiation 1992 ; 49 : 93-9.

10. Boncinelli E, Simeone A, Acampora D, Mavilio F. Hox gene activation by retinoic acid. Trends Genet 1991; 7 : 329-34.

11. Kessel M. Respecification of vertebral identities by retinoic acid. Development 1992 115 : 487-501.

12. Viallet JP, Ruberte E, Krust A, Zelent A, Dhouailly D. Expression of retinoic acid receptors and dermal-epidermal interactions during mouse skin morphogenesis. In Morriss-Kay G, ed. Retinoids in Normal Deve lopment and Teratogenesis Oxford : Oxford Publ 1992 : 199-210.

13. Hardy M. Glandular metaplasia of hai follicles and other responses to vitamin A excess in cultures of rodent skin. $J$ Embryol Exp Morphol 1968 ; 19 : 157-80.

14. Viallet JP, Ruberte E, Du Manoir S Krust A, Zelent A, Dhouailly D. Retinoic acid-induced glandular metaplasia in mouse skin is linked to the dermal expression of retinoic acid receptor $\beta$ mRNA. Dev Biol 1991 ; 144: 424-8.

15. Lumsden AGS. Spatial organization of the epithelium and the role of the neural crest cells in the initiation of the mammalian tooth germ. Development 1988 ; 103s : 155-69.
16. Dhouailly D. Specification of feather and scale patterns. In : Malacinsky GM, Bryant SV, eds. Pattern Formation. New York McMillan, 1984 : 581-601.

17. Sengel P, Pautou MP. Experimental conditions in which feather morphogenesis predominates over scale morphogenesis. Nature 1969 ; 222 : 693-4.

18. Dhouailly D, Hardy M, Sengel P. Formation of feathers on chick foot scales : a stage dependent morphogenetic response to retinoic acid. J Embryol Exp Morphol 1980 ; $58: 63-78$

19. Cadi R, Dhouailly D, Sengel P. Use of retinoic acid for the analysis of dermalepidermal interactions in the tarsometatarsa skin of the chick embryo. Dev Biol 1983 ; $100 ; 489-95$.

20. Brand NJ, Petkovich M, Krust A, et al. Identification of a second human retinoic acid receptor. Nature 1988 ; 332 : 850-3.

21. Krust A, Kastner P, Petkovich M, Zelent A Chambon P. A third human retinoic acid receptor. Proc Natl Acad Si USA 1989 ; 86 . 5310-4.

22. Petkovich M, Brabd NJ, Krust A, Chambon $\mathrm{P}$. A human retinoic acid receptor which belongs to the family of nuclear receptors. Nature 1987 ; 330 : 444-50.

23. Mauger A, Demarchez M, Herbage D, et al. Immunofluorescent localization of collagen types I and III, and of fibronectin during feather morphogenesis in the chicken embryo. Dev Biol 1982 ; 94 : 93-105.

24. Chuong CM, Edelman GM. Expression of ccll-adhesion molecules in embryonic induction. I Morphogenesis of nestling feathers. $J$ Cell Biol 1985 ; 101 : 1009-26.

25. Hirai Y, Nose A, Kobayashi S, Takeichi M. Expression and role of $\mathrm{E}-$ and $\mathrm{P}$ cadherin adhesion molecules in embryonic histogenesis. II Skin morphogenesis. Development 1989 ; $105: 271-7$.

26. Jones FS, Prediger EA, Bittner DA, De Robertis EM, Edelman GM. Cell adhesion molecules as targets for $H o x$ genes : neural cell adhesion molecule promoter activity is modulated by cotransfection with Hox-2.5 and 2.4. Proc Natl Acad Sci USA 1992 ; 89 : 2086-90.

27. Pelton RW, Saxena B, Jones M, Moses $\mathrm{HL}$, Gold LI. Immunohistochemical localization of TGF $\beta 1$, TGF $\beta 2$ and TGB $\beta 3$ in the mouse embryo : expression patterns suggest multiple roles during embryonic development. J Cell Biol 1991 ; 115 : 1091-105.

28. Duboule D. The vertebrate limb: a model system to study the Hox/HOM gene network during development and evolution. Bio Assays 1992 ; 14 : 375-83. 